\title{
MECHANICAL FAULT DIAGNOSIS USING A SVDD INTELLIGENT ALGORITHM
}

\author{
Ziying Zhang ${ }^{1}$ 2 $^{*}$, Xu Qian ${ }^{1}$ \\ ${ }^{1}$ China University of Mining and Technology, Beijing 100083, China; \\ 2Shanxi Institute of Energy, Taiyuan, 030006, China. \\ *Corresponding author: Ziying Zhang \\ Email: zhangziying29@126.com
}

\begin{abstract}
To shorten the time and improve the accuracy in mechanical fault diagnosis, the fault diagnosis mechanism is studied of the high voltage switch in power system, and a new system is proposed as a fast, safe, and accurate mechanical fault diagnosis scheme of high voltage switch. First, the basic structure and common faults of high voltage switches are analyzed, the feature data set has a poor classification effect, and the SVM (Support Vector Machine) algorithm model can process all the feature data for an effective classification and recognition process. Finally, the high voltage switches mechanical faults are extremely scattered, the improved SVDD (Support Vector Data Description) intelligent algorithm can acquire, screen, and classify the mechanical faults data. The results of the improved SVDD intelligent algorithm are less affected by the classification boundary and converge to the average sample value. The new fault diagnosis system is proposed for a high voltage switch based on the improved SVDD intelligent algorithm. Subsequently, the simulation experiment is conducted for a high voltage switch fault diagnosis system based on the SVDD intelligent algorithm. The experimental results show that compared with the traditional mechanical fault diagnosis system, the proposed system can identify new fault types effectively. Meanwhile, the proposed system can quickly and efficiently classify and identify the operation states and fault types of machinery. Consequently, the accuracy of the mechanical fault diagnosis system is improved, providing technical support for the power system operation. Hence, the proposed fault diagnosis system is very suitable for the production of the power system and can greatly promote mechanical fault diagnosis in China.
\end{abstract}

Keywords: Mechanical fault diagnosis; High voltage switch; SVM algorithm; SVDD algorithm.

\section{Introduction}

China has officially joined global industrialization since the Third Revolution in Scientific and technology. Then, China begins to industrialize rapidly and develops its high-techs, and gradually ranks among the world's great powers. The usage of industrial machinery in China is expanding, and the types are also increasing. The mechanical fault also increases with rapid industrialization, which poses a new problem. One of the tough issues is how to quickly deal with mechanical faults $[1,2]$. Here, the high voltage switch with complex structure and difficult fault diagnosis ability is selected to study the mechanical fault diagnosis system. The high voltage switch is one of the crucial pieces of equipment in the power system and can control the electrical switch, allocate electrical load, and operate electrical machinery $[3,4]$. When the electrical machinery fails, the high voltage switch can disconnect the electrical equipment timely, facilitating the relevant personnel to diagnose the fault.
Thus, the fault-free parts can operate smoothly, and the faults can be solved in time so that the electrical machinery can quickly return to its normal state with lesser influence $[5,6]$. The internal structure of a high voltage switch is rather complex, and it is often exposed to very harsh environmental conditions, leading to frequent faults $[7,8]$.

Here, the fault types and diagnosis algorithms are studied of the $12 \mathrm{KW}$ high voltage switch. Then, a fault diagnosis system is proposed of high voltage switch based on SVDD (support vector data description) intelligent algorithm. First, the basic structure and common fault types of high voltage switches are analyzed, and the methods of acquiring fault data based on mechanical vibration signals are summarized. Afterward, the SVM (Support Vector Machine) algorithm is analyzed on its concepts and limitations, and the basic principle and operation process of the improved SVDD algorithm are deduced.

Finally, a new fault diagnosis system of high voltage switch is designed and simulated. 


\section{Method}

\subsection{Basic Structure and Common Faults of the High Voltage Switch}

\section{Basic structure of high voltage switch}

The performance of a high voltage switch depends on its internal arc-control efficiency. High voltage switch can be divided into oil switch, vacuum switch, sulfur hexafluoride switch, air switch, and magnetic switch according to the arc-control types.
A high voltage switch should be controllable during operation to move the contact parts quickly. There are various ways to control switches, such as spring control, hydraulic control, pneumatic control, electromagnetic control, and permanent magnet control. Although the high voltage switches differ in type, control mode, and operating conditions, they are composed of the control part, the transmission department, the electric switch, the external insulation component, and the pedestal, as shown in figure 1.

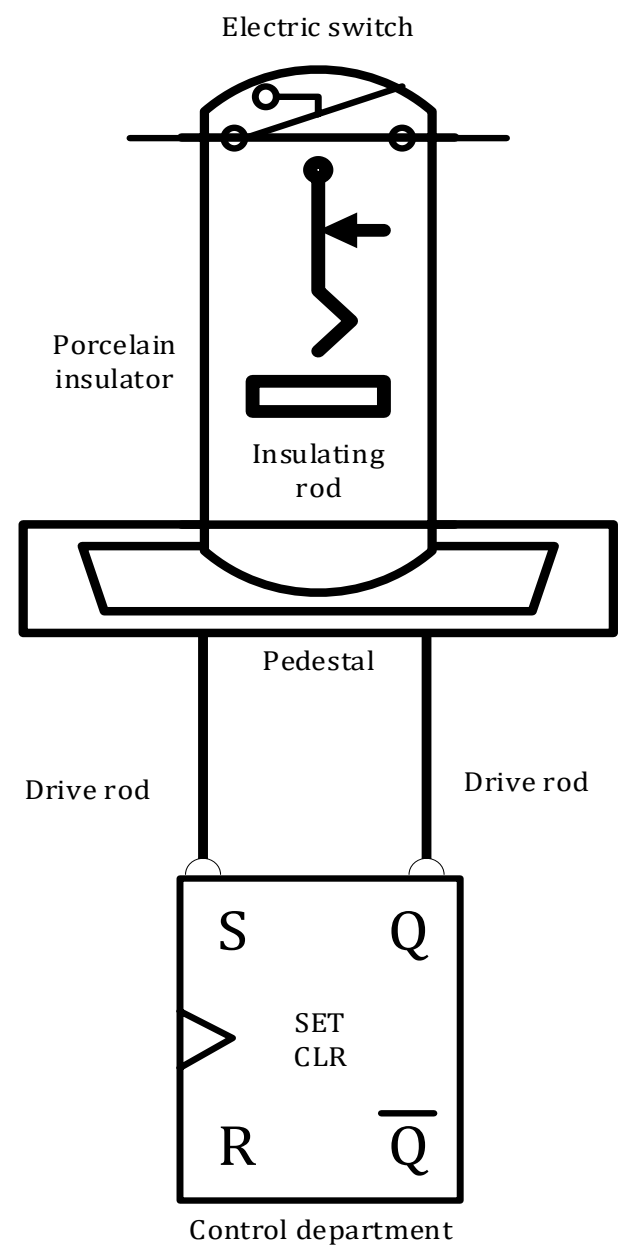

Figure 1: Basic structure of high voltage switch

The components and main functions of high voltage switches are analyzed next.

(1) Control department: it controls the entire mechanical rotary system and electric switch.

(2) Transmission department: it is composed of conveyors and gear and transmits energy to the gate switch.

(3) Insulation external part: it is composed of a porcelain insulator and supports high voltage switches. When a fault occurs, it is timely insulated from gear.

(4) Electric switch: it opens or closes the open circuit and is composed of contact parts and an arccontrol device.
(5) Pedestal: it is the base of the whole high voltage switch and can support and fix machinery.

\section{Common faults of the high voltage switch}

The high voltage switch is composed of a control mechanism, transmission mechanism, and upload mechanism.

The structure of the transmission and upload mechanism is simple with few fault occurrences. Thus, the mechanical faults of the high voltage switch are mostly from the control part $[9,10]$, as shown in figure 2. 


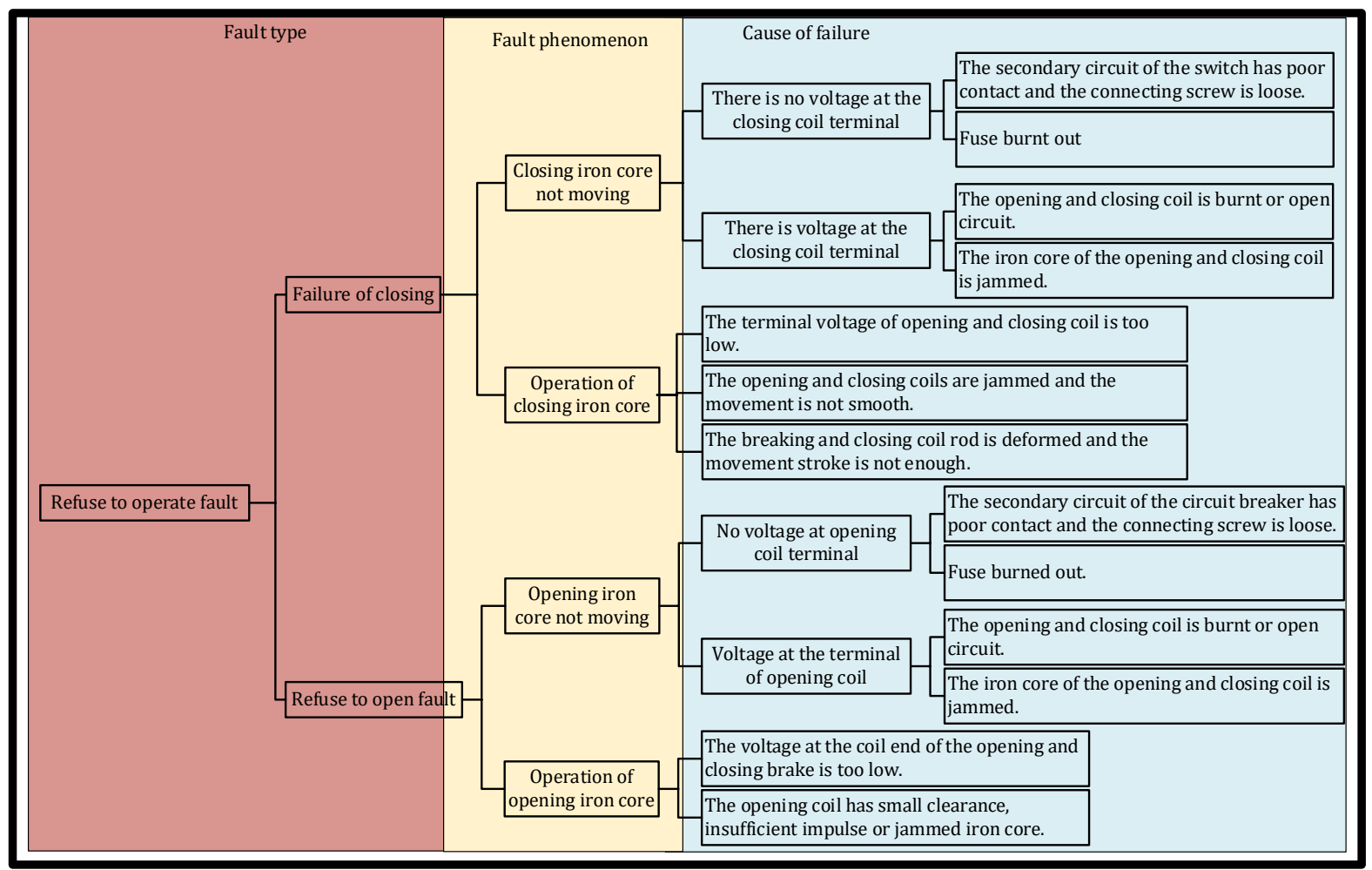

(a) Failure of high voltage switch and its causes

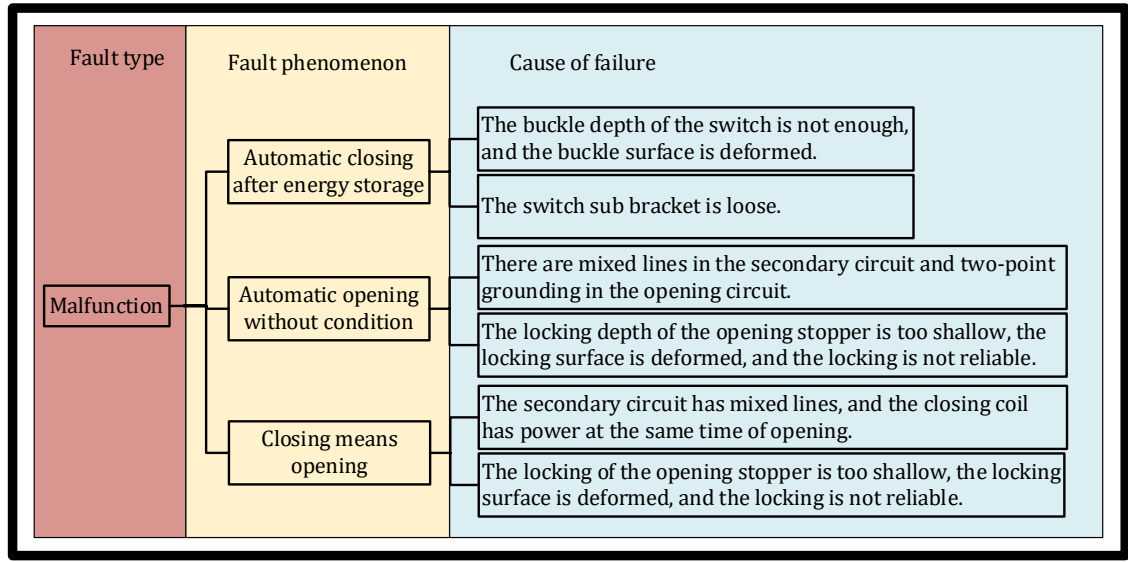

(b) Malfunction of high voltage switch and its causes

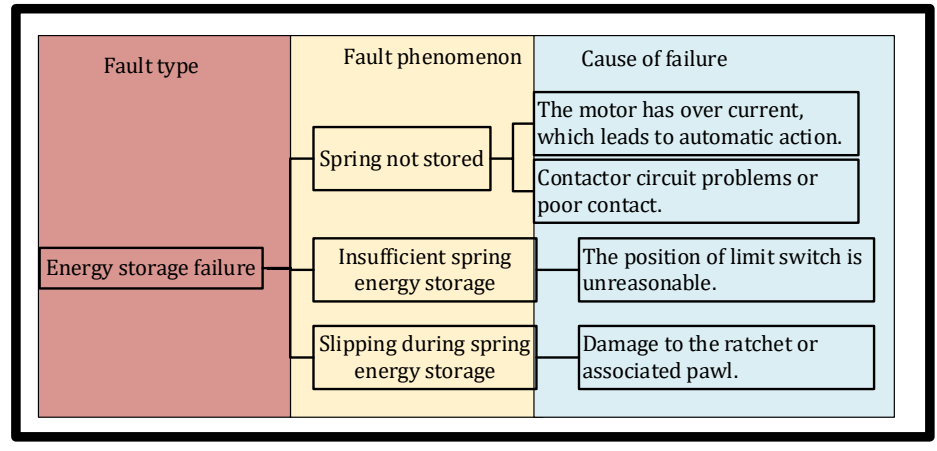

(c) Energy storage failure of high voltage switch and its causes

Figure 2: Types, phenomena, and causes of faults in high voltage switch control unit

Figure 2 shows that the causes of mechanical faults of high voltage switch can be attributed to the following points: the switch gate is jammed, the equipment position is improper, the parts are prone to fall off, or the size is not matched. Though hydraulic control is barely used today, its fault types, 
such as the valve rod control fault, the hydraulic insufficiency, and the transportation pipe jamming, should be considered. The phenomena and causes of high voltage switch faults are summarized here. The results show that the mechanical faults of high voltage switch can be roughly divided into refuse to operate fault, malfunction fault, and energy storage failure. Meanwhile, the causes can be summarized as the following three points. First, the internal parts may be displaced, deformed, and damaged. Second, the contact closing is easy to loosen or jam. Third, the pin roll loosens easily.

\subsection{Acquisition of Mechanical Control Signal of the High Voltage Switch}

\section{Control signal acquisition method for a high} voltage switch

At present, most of the domestic high voltage switch signals are based on mechanical vibration, and the acceleration vibration sensor is adopted for signal acquisition. The acceleration vibration sensor is the best processor for high voltage and strong magnetic field because of its advantages, including lightweight, small size, less noise, and good antiinterference effect. Besides, it takes less time to adapt to the working environment [11]. Its structure is shown in figure 3.

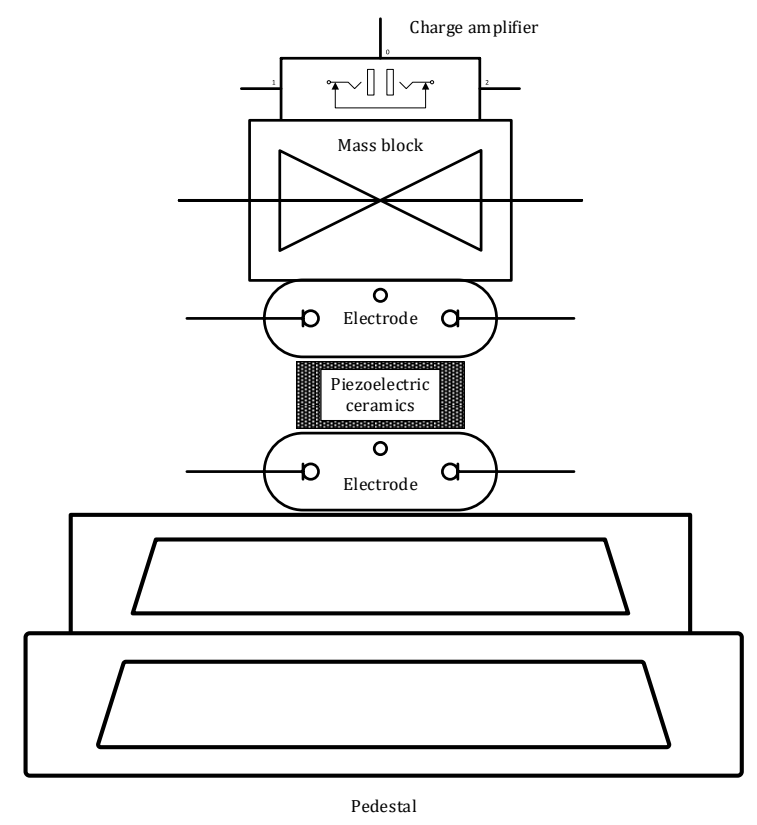

Figure 3: Structure of acceleration vibration sensor

The way the acceleration vibration sensor is installed affects the acquisition of control data, because the anti-interference ability of the sensor is different under different installation methods, and the acquired control signal is also different. The pedestal of the sensor must be fully contacted with the high voltage switch and rigidly fixed on installation. The sensor is installed through the magnetic adsorption method. The installation position is crucial for the acceleration vibration sensor. Generally, the sensor should be installed as near the high voltage switch control source as possible. Thus, the sensor should be installed near the conveyor to acquire control signals.

2. Control signal extraction method of the high voltage switch

Due to the complexity and variety of control signals in high voltage switches, the control signals in different states are separated through the WPD (Wavelet Packet Decomposition) sub-band tree transformation method. Consequently, the control information of the high voltage switch can be better grasped. The schematic diagram is shown in figure 4 .

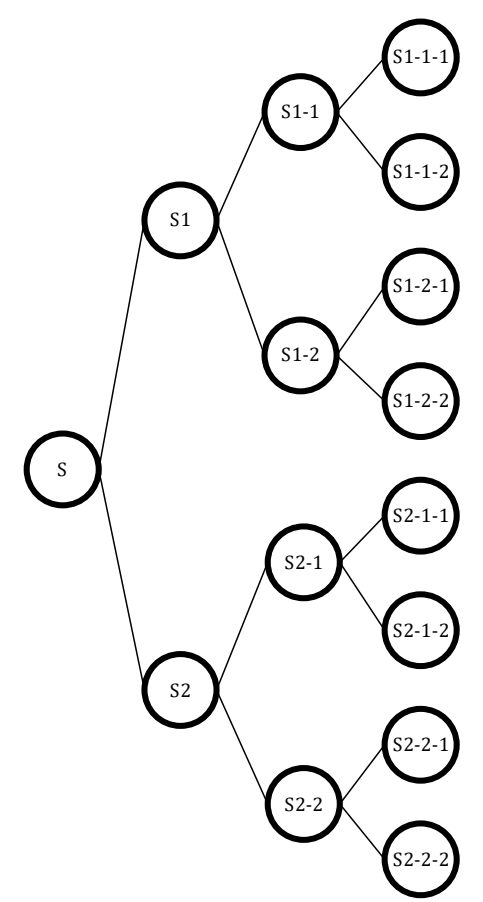

Figure 4: WPD schematic diagram

The inspiration of the WPD method comes from the analysis tree, and the data iteration is converted into different levels of content through layer-bylayer separation [12]. The methods have been developed from sub-band tree analysis. Besides subband tree analysis features, the WPD can also analyze data systems with high and low-frequency control signals.

The fault control signal of the high voltage switch is unstable and nonlinear. The WPD method can analyze different kinds of control signals comprehensively and accurately, so it is often applied to fault diagnosis of electrical equipment.

\subsection{SVM Algorithm}

The SVM (Support Vector Machine) can map the unclassifiable factors in the low dimension space to the high dimension space through the kernel 
function. Meanwhile, the farthest hyperplane from the sample in the high dimension space can classify and process all data in detail. Consequently, the generalization ability of the classifier to the new sample content is strengthened, while the confidence risk is reduced. Hence, the SVM can minimize the operational risk and identify and analyze different data samples [13].

To improve classification and reduce noise influence, the relaxation factor is included in the SVM algorithm to adjust the classification and collation of information in the hyperplane. The new SVM algorithm function is expressed in equation (1).

$$
\left\{\begin{array}{l}
\min \frac{1}{2}\|\mathrm{u}\|^{2}+\mathrm{P} \sum_{a=1}^{b} \Omega_{a} \\
\Omega \geq 0, a=1,2 \ldots \mathrm{n}
\end{array}\right.
$$

In equation (1), $\min _{2}^{\frac{1}{2}\|u\|^{2}}$ expresses the classification interval, $\Omega_{a}$ denotes the relaxation factor, and $P$ represents the penalty factor. The purpose is to find the best equilibrium point between the maximum interval and the sample.

\subsection{SVDD Algorithm}

SVDD (Support Vector Data Description) algorithm is a single classification algorithm derived from SVM. Compared with the SVM algorithm, the SVDD algorithm is can study small data sets and can process nonlinear data efficiently. The SVDD algorithm can classify unbalanced data [14].

The objective function of the standard SVDD algorithm is shown in equation (2).

$$
R^{2}+P \sum_{b=1}^{a} \mu_{b}^{2}
$$

In equation (2), $R$ denotes the radius of the information sphere, $P$ expresses the penalty parameter, and $\mu_{\mathrm{b}}{ }^{2}$ represents the relaxation variable. The objective function of the improved SVDD is as follows.

$$
\begin{aligned}
& \min _{R^{2}}+P \sum_{b=1}^{a} \mu_{b}^{2} \\
& \text { s.t. }\left\|\theta\left(y_{b}\right)-o\right\|^{2} \leq R^{2}+\mu_{b}^{2}
\end{aligned}
$$

In equations (3) and (4), $o$ is a column vector representing the center of the circle, and $\theta\left(y_{b}\right)$ denotes a feature function that maps data from the input space to the feature space distance. $P$ stands for a penalty factor. The greater the $P$, the greater the penalty for error type discrimination [15].

The radius of the sample circle and the number of samples it contains need to be adjusted through parameters setting. If the number of samples is large, the sample is put into the circle as far as possible; if the number of samples is small, the area of the circle needs to be reduced.

The improved SVDD algorithm does not need the constraint condition $\mu_{b} \geq 0, b=1,2,3 \ldots n$. If several $b$ s can be derived from $\mu_{b}<0$ and $\mu_{b}{ }^{*}=0$, the following conditions are satisfied $[16,17]$.

$$
\left\|\theta\left(y_{b}\right)-o\right\|^{2} \leq R^{2}+\mu_{b}^{*} \leq R^{2}+0
$$

Under this condition, the target value will get smaller and smaller, and $\mu_{b}$ will never be the optimal solution.

Then, the Lagrange multiplier $\beta b$ is introduced, and the Lagrange functions in equations (4) and (5) will be transformed into the following equation.

$$
L=R^{2}+\sum_{b=1}^{a} \mu_{b}^{2}-\sum_{b=1}^{a} \beta_{b}\left(R^{2}+\mu_{b}-\left\|\theta\left(y_{b}-o\right)\right\|^{2}\right)(6)
$$

In equation (6), if partial derivatives of $L$ from $o$, $\mu_{b}$, and $R$, equal zero, the following equations can be derived.

$$
\begin{aligned}
& \frac{\Omega L}{\Omega R}=2 R-2 R \sum_{b=1}^{a} o_{1}=0 \Rightarrow \sum_{b=1}^{a} o_{1}=1 \\
& \frac{\Omega L}{\Omega o}=-2 R \sum_{b=1}^{a} o_{1}\left(\theta\left(\mathrm{y}_{b}\right)-o\right)=0 \Rightarrow o \Rightarrow \sum_{b=1}^{a} \beta_{b}\left(\theta_{b}\left(\mathrm{y}_{b}\right)\right) \\
& \frac{\Omega L}{\Omega \mu_{b}}=2 P \mu_{b}-\theta_{b}=0 \Rightarrow \theta_{b}=2 P \mu_{b}
\end{aligned}
$$

The dual problem of the optimization problem can be obtained when equations (7), (8), and (9) are introduced into the Lagrange function in equation (6).

$$
\begin{aligned}
\max L= & \sum_{b, c=1}^{a} \beta_{b} P\left(y_{b}, y_{c}\right)-\sum_{b, c=1}^{a} \beta_{b} \beta_{c}\left(P\left(y_{b}, y_{c}\right)+\frac{1}{4 P} \mu_{b c}\right) \\
& \text { s.t. } \sum_{b=1}^{a} \beta_{b}=1, b=1,2 \ldots \mathrm{n} \\
& 0 \leq \beta_{\mathrm{b}}, \quad b=1,2 \ldots \mathrm{n}
\end{aligned}
$$

In the above equations, the value $\mu_{\mathrm{bc}}$ is shown below.

$$
\mu_{\mathrm{bc}}\left\{\begin{array}{l}
1, \quad b=c \\
0, a \neq c
\end{array}\right.
$$

The above derivations show that the SVDD operation directly affects the quadratic term of the objective function and the constraint operation of the dual problem.

Consequently, the SVDD algorithm is improved to reduce the loss deviation of a quadratic function to quadratic through the penalty factor. This is another form of SVDD algorithm under the Lagrange function. Based on the above optimization, the improved SVDD algorithm is as follows. 


$$
\begin{aligned}
& \beta_{b}\left(R^{2}+\mu_{b}-\left\|\theta\left(y_{b}\right)-o\right\|^{2}\right)=0 \\
& \left\|\theta\left(y_{b}\right)-o\right\|^{2} \leq R^{2}+\mu_{b}, \beta_{b}=1,2, \ldots \mathrm{n}
\end{aligned}
$$

\subsection{Design of Mechanical Fault Diagnosis Model for a High Voltage Switch}

The control signal can reflect the operation states of a high voltage switch, and the eigenvector can express the operation states through analysis. Consequently, a fault diagnosis system is proposed of high voltage switch based on SVDD intelligent algorithm.

The acceleration vibration piercer.... can acquire the control signal of the high voltage switch, and the SVDD algorithm can identify, classify, and analyze the eigenvectors.

The flowchart is shown in figure 5 .

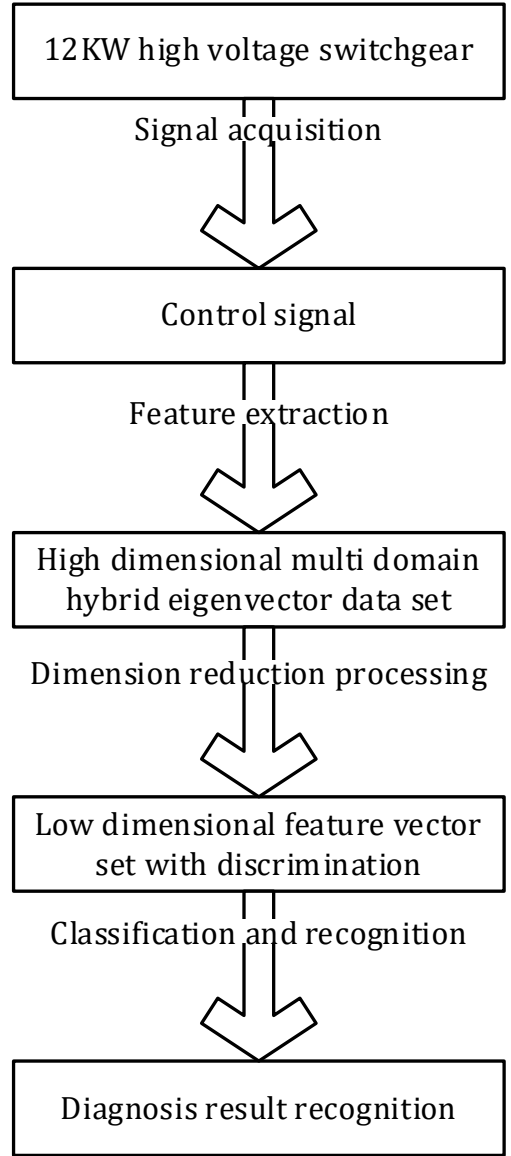

Figure 5: Flowchart of mechanical fault diagnosis for a high voltage switch

Subsequently, a high voltage switches mechanical diagnosis model is proposed based on the SVDD algorithm to solve problems, such as small sample, unbalanced, and nonlinear operation. The operation flow of the proposed model is as follows.

(1) The control signal of the high voltage switch with 2000 sampling points is sorted by time, and the data set $G \in R^{\mathrm{G}^{*} 2000}$ with $G$ samples is obtained.

(2) Then, each control signal sample in $G \in R^{\mathrm{G}^{*} 2000}$ is decomposed through the sub-band tree decomposition rule. The small and medium functions of the sub-band tree rule are decomposed into the $M$ layer through the DB2 function, and $2^{\mathrm{m}}$ sub-band tree nodes can be obtained.
The coefficient set of the sub-band tree nodes is denoted as $E \in R^{\left(2^{m} * W P N_{m}\right) * G}$, and $\mathrm{WPN}_{\mathrm{m}}=2000 / 2^{\mathrm{m}}$ represents the number of coefficients of the sub-band tree node.

(3) The values of EWPN and REWPN of each subband tree node in the set $E \epsilon R^{\left(2^{m * W P N} N_{m}\right) * G}$ are obtained to represent eigenvectors. Then, the feature set $Y \in R^{2^{m_{*} G}}$ of the training-samples are obtained. $Y=\left[y_{1}, y_{2}, \ldots y_{\mathrm{n}}\right]$ denotes a $2^{m} * G$ matrix, $G$ represents the number of samples, and the data are concentrated in the following circles.

$$
y_{n}=\left[E W P N\left(t_{1}\right), \ldots, \operatorname{EWPN}\left(t_{2^{\mathrm{m}}}\right), \operatorname{REWPN}(t), \ldots, \quad R E W P N\left(t_{2^{\mathrm{m}}}\right)\right]^{G} t=1,2, \ldots, G
$$

In equation (14), $y_{n}$ represents any sample. 
(4) Subsequently, the feature set $F=[F 1, F 2, \ldots F n]$ obtained through step (3) is processed with principal component analysis. The high-dimensional features are projected into the low-dimensional space through a spatial vector, and then the lowdimensional feature set $Y_{R E V N} \in R^{d \times G}$ is obtained.

(5) The sample set $Y_{R E V N}$ is trained through the SVDD algorithm classifier.
(6) A new sample set must go through the above steps (1)-(5) for the feature set $Y_{R E V N}^{\prime} \in R^{d \times 1}$. Then, the feature set is input into the SVDD algorithm classifier to identify and analyze the control signals. The calculation results of the classifier can diagnose the high voltage switch [1820]. The calculation model is shown in figure 6.

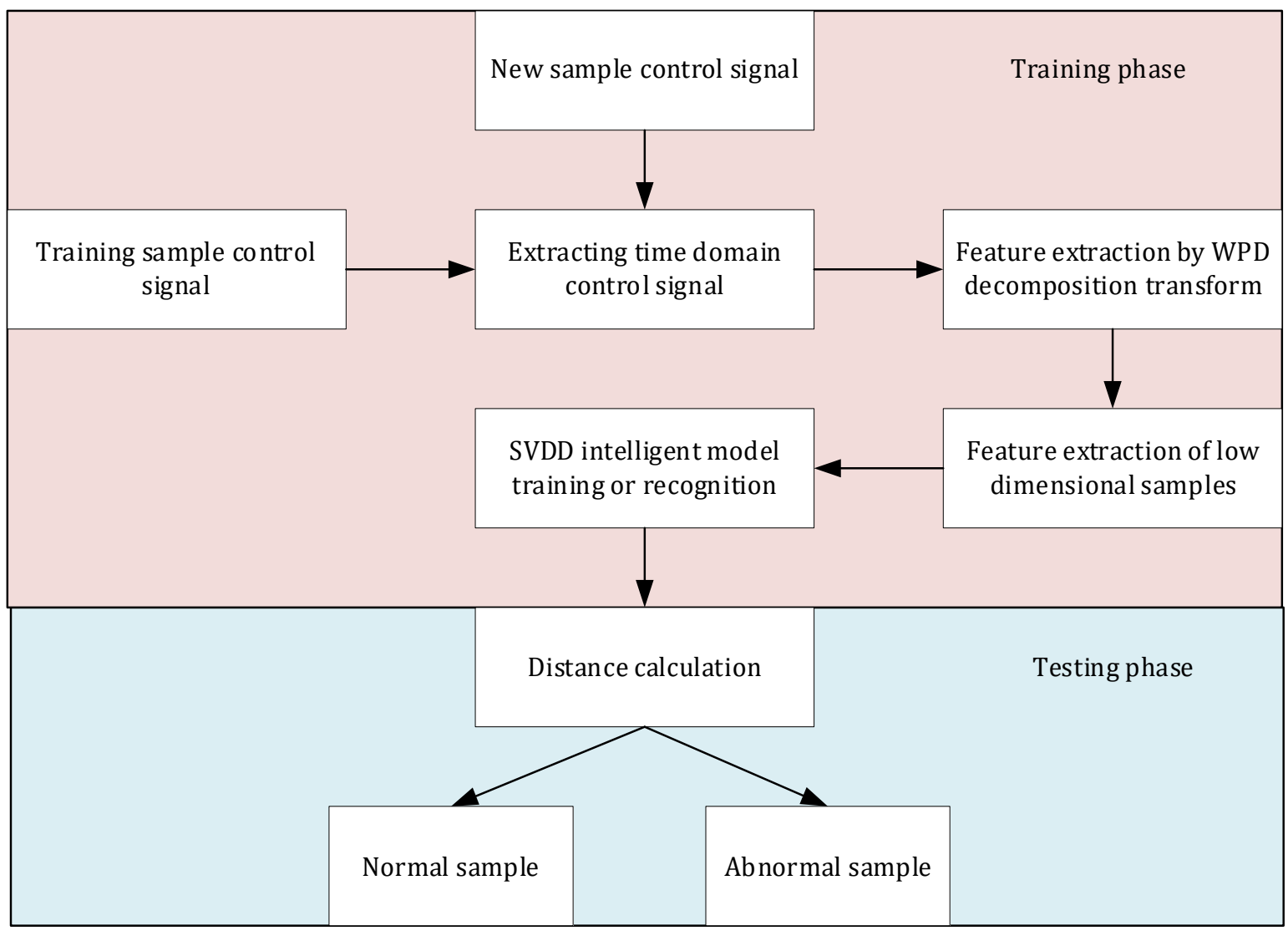

Figure 6: Flowchart of mechanical fault diagnosis for a high voltage switch

Figure 6 shows the characteristics of a mechanical fault diagnosis system based on the SVDD algorithm [21].

(1) First, the WPD method can obtain the highdimensional eigenvectors, and the principal component analysis can reduce the dimension of the high dimensional eigenvector samples that are input into the SVDD classifier. Consequently, the sample set shows low-dimensional features and has discrimination, and the overall initial structure is stable. These low dimension samples set can reduce the operation time of the classifier, improve the operation efficiency, and enhance the classifier's ability to analyze and process eigenvectors.

(2) The SVDD intelligent algorithm is used as a fault diagnosis classifier for high voltage switches. The fault diagnosis system is improved based on the classification algorithm, and the detection efficiency is greatly improved.

\subsection{Parameter Setting of the Simulation Experiment}

The parameters of the SVDD intelligent algorithm are selected and analyzed. The optimal kernel radius of the SVDD intelligent algorithm is set as $r=0.5$, the penalty factors are set as $P 1=10$ and $P 2=1$. Then, the classifier design is completed. The evaluation index should be determined to evaluate the mechanical fault diagnosis model of high voltage switch.

The evaluation index of a single effect algorithm cannot analyze comprehensively since the accuracy is largely affected by the recall rate. Thus, the evaluation index that combined recall rate and accuracy is adopted to comprehensively evaluate the recognition and analysis ability of the model. Finally, the F-Measure weighted comprehensive evaluation index is chosen [22]. 


\section{Results and Discussion}

\subsection{Evaluation of Mechanical Fault Diagnosis} Model for a High Voltage Switch

Three types of fault samples, including iron core jam fault, base looseness fault and poor lubrication fault are mixed into the normal samples and analyzed together to evaluate the mechanical fault diagnosis model of high voltage switch.
To prove the superiority of the proposed model, the dimensions are reduced off the KECA algorithm, PCA algorithm, and KPCA algorithm.

Subsequently, different types of low dimension eigenvectors are obtained and then are input into the SVDD classifier [23].

Their difference in F-measure performance is shown in figure 7.

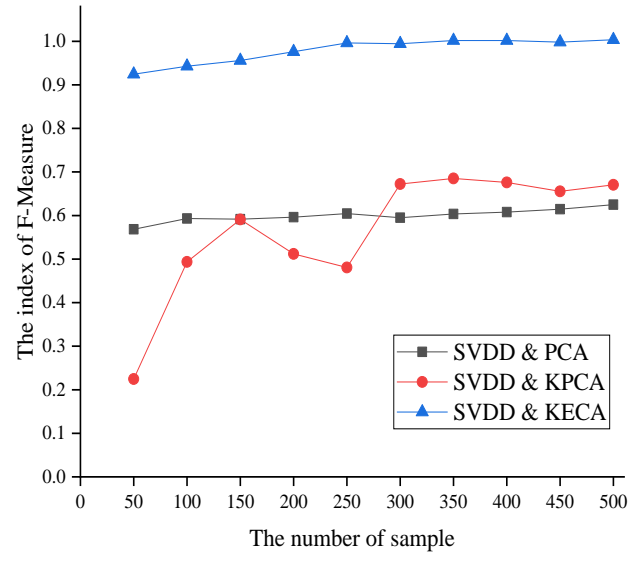

(a) F-measure performance curve of mechanical jam fault of high voltage switch

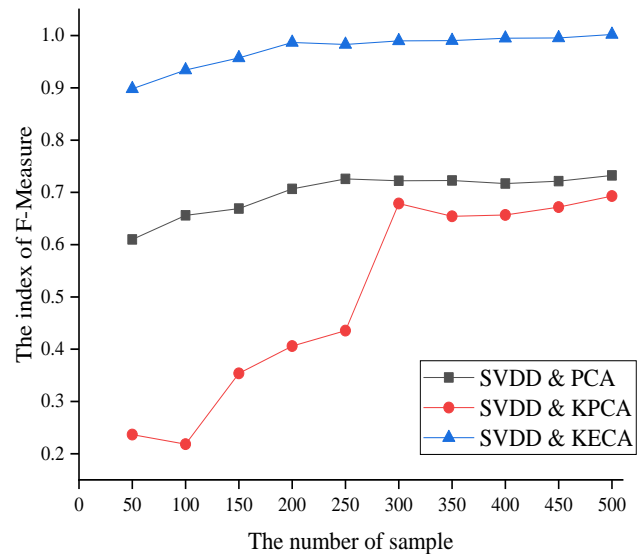

(b) F-measure performance curve of high voltage switch base looseness fault

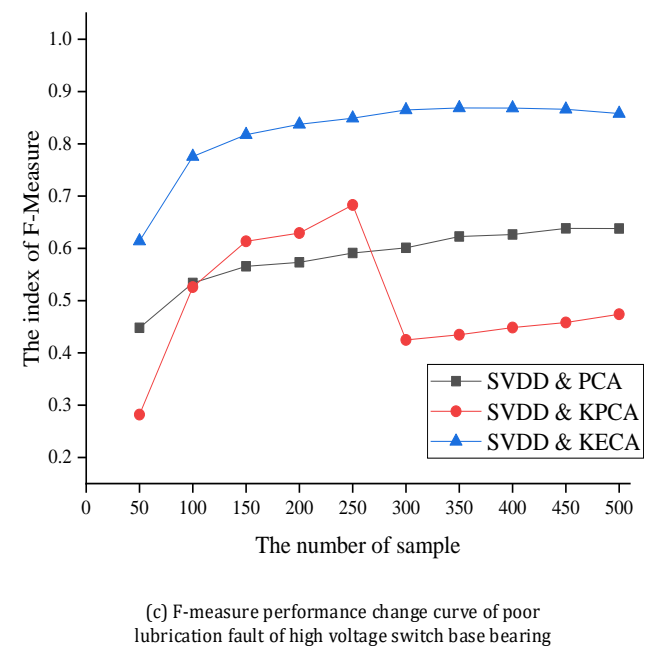

Figure 7: F-Measure performance curves of three fault types

Figure 7 shows that the F-Measure performance curve of the high voltage switch mechanical fault diagnosis model based on the SVDD intelligent algorithm is much higher than that of the traditional fault diagnosis model. Meanwhile, the F-Measure curve always remains stable at a high level and not susceptible to unbalanced environmental factors.

Accordingly, the proposed model has high adaptation and accuracy for the diagnosis of high voltage switch mechanical faults can always maintain good generalization performance and is hardly influenced by unbalanced factors. Thus, the SVDD intelligent algorithm is suitable for the fault diagnosis of high voltage switches [24].

The average F-Measure values of 500 poor lubrication faults of bearing in high voltage switch are compared and analyzed to evaluate the proposed fault diagnosis model, as shown in figure 8. 


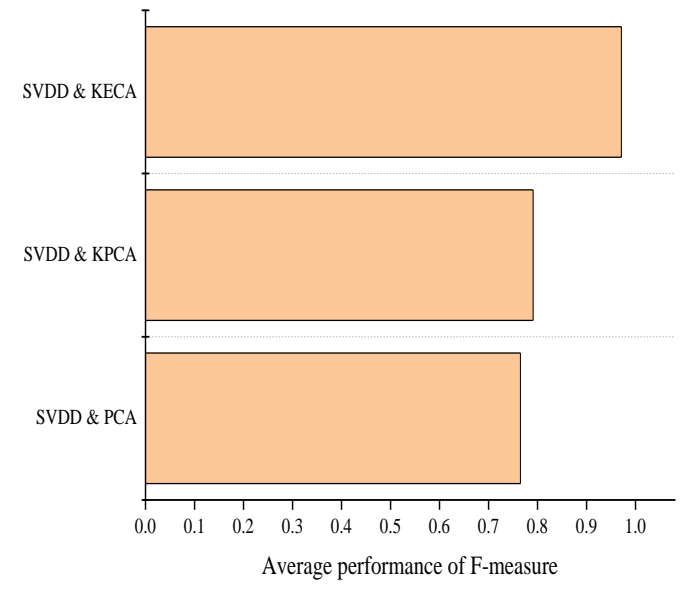

Figure. 8 Average F-Measure performance of poor lubrication fault samples of high voltage switch bearings with three different models

Figure 8 indicates that the mechanical fault diagnosis model based on SVDD can retain the initial structure of the original data so that the sample has obvious distinguishing features after dimension reduction operation. Consequently, the classification process has become more convenient.
By contrast, other similar model algorithms can only reduce the dimension of the data and cannot automatically output the data set of low-dimensional spatial features. Meanwhile, the model features change dramatically, the average value is medium, and the generalization performance is insufficient. Hence, the F-Measure classification model has a much higher performance than the other similar models [25].

\subsection{The Results and Analysis of the Mechanical Fault Diagnosis System of the High Voltage Switch}

The different state features set obtained through dimension reduction are input into the SVDD intelligent algorithm classifier to simulate and evaluate the mechanical diagnosis model of high voltage switch. 800 samples are extracted from each of the four control signals to form a set, and 400 training sample sets together with 400 diagnostic sample sets are established. Then, four groups of test samples are chosen from the diagnostic sample sets with each group containing 200 test samples. The samples' states are shown in figure 9.

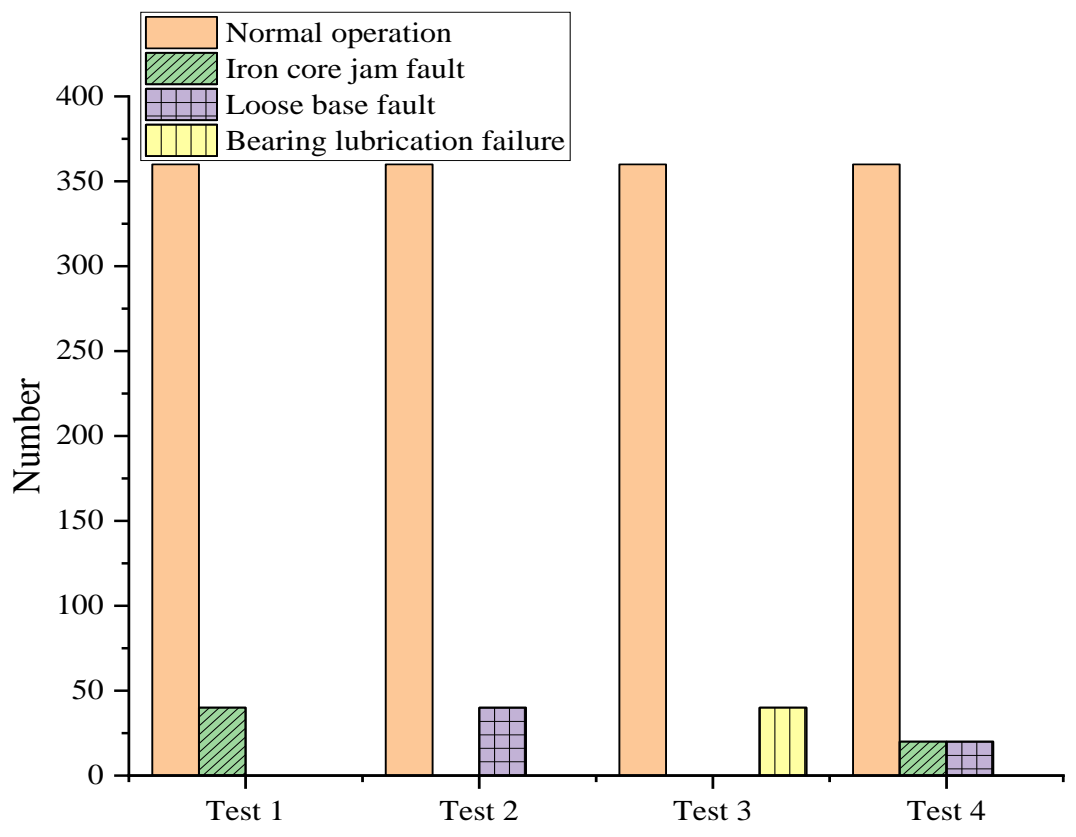

Figure 9: Test sample state statistics

The high voltage switch mechanical fault diagnosis model is simulated and analyzed for a straightforward and visualized effect. SVDD intelligent algorithm, SVDD mechanical algorithm, and SVM algorithm can conduct the same simulation experiment on the features in the classifier. SVDD mechanical algorithm and SVM algorithm are based on the optimal parameters.
Then, the average accuracy is counted of SVDD intelligent algorithm, SVDD mechanical algorithm, and SVM algorithm for different test data samples and used as an index for the ratio of the correct sample to the total sample number. which is regarded as the classification accuracy. The fault diagnosis results of the above three algorithms are shown in figure 10. 


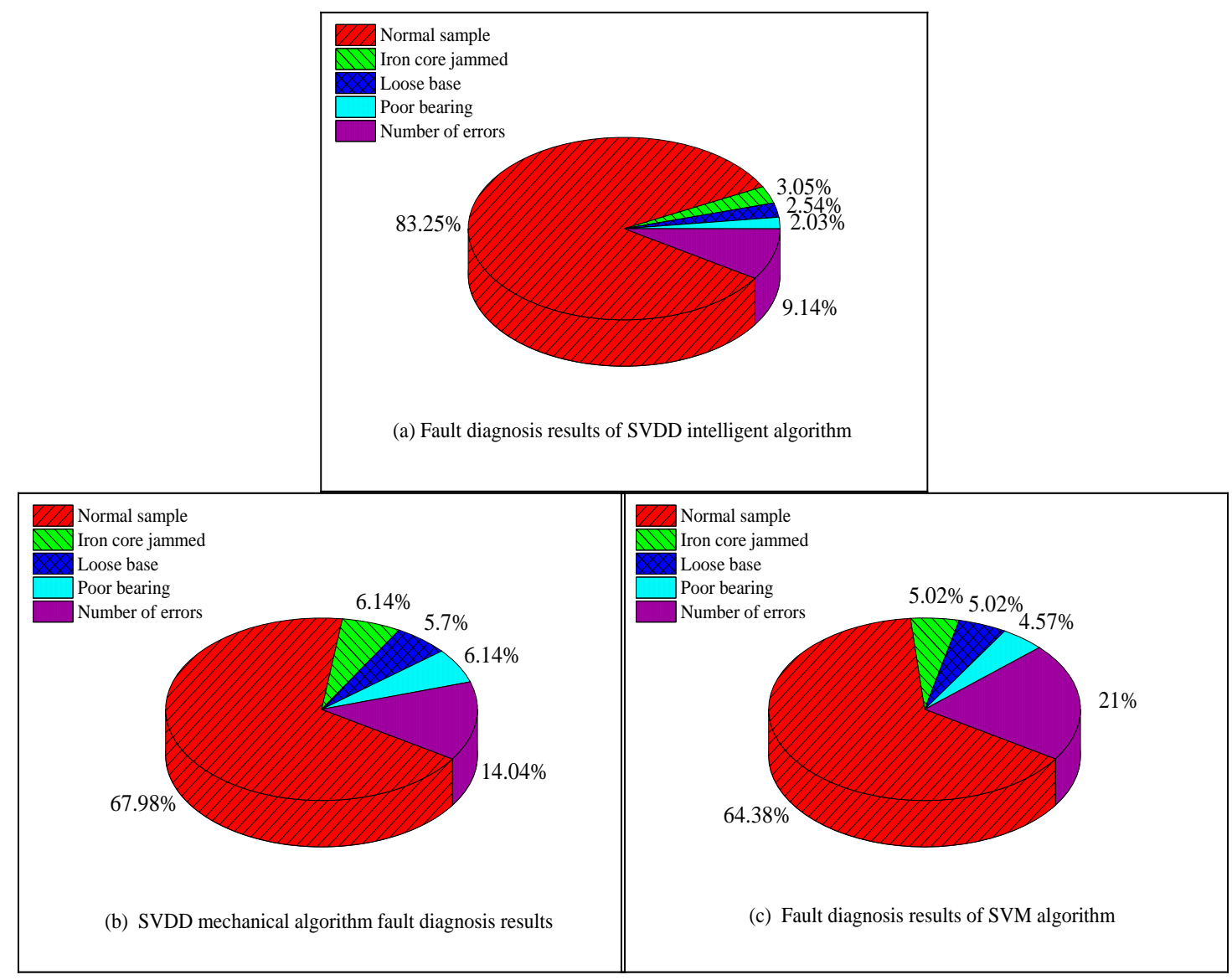

Figure 10: Mechanical fault diagnosis results of high voltage switch under three algorithms

Figure 10 shows that the accuracy of the SVDD intelligent algorithm is much higher than that of the SVDD mechanical algorithm and SVM algorithm for different types of fault samples of high voltage switch. The accuracy of the SVDD intelligent algorithm is $83.25 \%$, and in the simulation experiment, no known fault types are identified as unknown faults through the SVDD intelligent algorithm. Meanwhile, the recognition error rate is low. By contrast, the SVDD mechanical algorithm and SVM algorithm occasionally identify known faults into unknown faults and have low classification accuracy. Thus, the SVDD intelligent algorithm-based fault diagnosis model has the highest accuracy and suitable for high voltage switch mechanical production.

\section{Conclusions}

The high voltage switch in the power system is analyzed to solve the problems in the traditional mechanical fault diagnosis system, such as low efficiency, low detection accuracy, and the inability to detect new faults, and then the fault diagnosis feature analysis method and classification algorithm are studied. Finally, the high voltage switch fault diagnosis system is established based on SVDD intelligent algorithm.
First, the structure and common mechanical faults are analyzed and summarized of the high voltage switch. The acceleration vibration sensor is selected to acquire the control signal, and then the high-dimensional eigenvectors of the high voltage switch operation state are extracted through the sub-band tree method. Afterward, a mechanical fault diagnosis classifier based on SVDD intelligent algorithm is proposed due to the unbalanced data acquisition of high voltage switch mechanical fault data. Consequently, the recognition range of the classifier is expanded, and the classification accuracy is improved. Finally, a mechanical fault diagnosis system of high voltage switch based on SVDD intelligent algorithm is established, and the classifier under this algorithm is compared with the classifiers of other algorithms. The results show that SVDD intelligent algorithm can effectively diagnose the mechanical faults of high voltage switch, and the limitations of traditional methods are solved, such as high dependence on sample data, unable to identify unknown faults. Hence, the accuracy and efficiency are improved of mechanical fault diagnosis of high voltage switch.

There are still some deficiencies, including the following two points. (1) It is hoped that further studies are needed to explore how to improve the performance of the SVDD algorithm without considering samples. (2) Internet technology should 
be utilized in future research to design fault diagnosis with network and online features. The high voltage switch with a real-time monitoring network is believed to be the direction of future research for mechanical fault diagnosis improvement.

\section{References}

[1] Cheng T.T., Gao W.S., Liu W.D., et al. (2018) Evaluation method of contact erosion for high voltage SF6 circuit breakers using dynamic contact resistance measurement. Electric Power Systems Research, 163(10), 725-732.

[2] Li M.Q., Yang R.W., Zhang H., et al. (2019) Development of a flavor fingerprint by HS-GC-IMS with PCA for volatile compounds of Tricholoma matsutake Singer. Food Chemistry, 290(8), 32-39.

[3] Cai Z.Y., Zhang M., Bao S.S. (2019) Interval based fault diagnosis method for batch process based on KECA and FWA-SVM. Application Research of Computers, 36(5), 1409-1414.

[4] Qin X.Q., Liu D., Wang D., (2019) Social relationships classification using social contextual features and SVDD-based metric learning. Applied Soft Computing. 77(4), 344-355.

[5] Shereen A., Hamid G.H., Roopak S. (2019) A system on chip for melano ma detection using FPGA-based SVM classifier. Microprocessors and Microsyste ms.65(3), 57-68.

[6] Liu Y.F., Jiang B., Yi H. (2019) Transformer fault diagnosis method based on slrmd and multi class SVM. Machine Design and Manufacturing Engineering. 48(12), 71-75.

[7] Wang S., Minku L.L., Yao X. (2015) Resamplingbased ensemble methods for online class imbalance learning. IEEE Trans. Knowledge Data Engineering. 27(5), 1356-1368.

[8] Liu C.F., Cui H.Y., Li X., et al. (2019) Transformer fault diagnosis based on support vector machine under asymmetric samples. High Voltage Apparatus, 55(07), 216-220.

[9] Huang N.T., Chen H.J., Lin L., et, al. (2018) Mechanical Fault Diagnosis of High Voltage Circuit Breakers Based on S-transform and Extreme Learning Machine. High Voltage Apparatus, 54(6), 74-80.

[10] Han Y., Dong B. (2019) A mechanical fault diagnosis method of high voltage circuit breaker based on coil current. High Voltage Apparatus, 55(9), 241-246.

[11] Li C., Zhou J.P., Wan S.T., et al. (2019) Mechanical Fault Diagnosis of High Voltage Circuit Breakers Based on Current Characteristics of Switching Coil. Zhejiang Electric Power, 38(1), 98-103.

[12] Jin X.C., Cui H.S., Wu J.W., et al. (2019) Fault diagnosis of circuit breaker based on wavelet packet energy spectrum and delay time. High Voltage Apparatus, 66(6), 38-44.
[13] Peng Z.X., Wang S., Yi L., et al. (2019) Research on fault diagnosis of high voltage circuit breaker opening and closing coil based on SVM principal component analysis. High Voltage Apparatus, 55(7), 39-46.

[14] Lin L., Chen Z.Y. (2019) Fault Diagnosis of Operating Mechanism of High-voltage Circuit Breaker Based on Fuzzy RBF Neural Network. High Voltage Apparatus, 55(10), 52-58.

[15] Seongmin H., Jay H.L. (2018) Fault detection and classification using artificial neural networks. IFAC-Papers OnLine. 51(18), 470-475.

[16] Liu R.H., Dou L.J., Wan S.T., et al. (2018) Fault Diagnosis of High Voltage Circuit Breaker Based on EEMD Sample Entropy and Support Vector Machine. Journal of North China Electric Power University, 45(2), 82-88.

[17] Zhou Q.C., Liu X.C., Zhao J. (2018) Research on one dimensional deep convolution neural network fault diagnosis of rotating machinery. Journal of Vibration and Shock, 37(23), 31-37.

[18] Hadi S. (2020) Fault diagnosis of nonlinear systems using recurrent neural networks. Chemical Engineering Research and Design, 153(1), 233-245.

[19] Seongmin H., Jay H.L. (2018) Fault detection and classification using artificial neural networks. IFAC-PapersOnLine.51(18), 470-475.

[20] Li Y.F., Wu C.P., Tu X. (2019) Fault diagnosis method of vibration sensor based on PCA and SVM. Process Automation Instrumentation, 40(10), 48-52.

[21] Tang X.Z., Hong H.Y., Shu Y.Q., et al. (2019) Urban waterlogging susceptibility assessment based on a PSO-SVM method using a novel repeatedly random sampling idea to select negative samples. Journal of Hydrology, 576(9), 583-595.

[22] Li W., Peng M.J., Wang Q.Z. (2018) Fault detectability analysis in PCA method during condition monitoring of sensors in a nuclear power plant. Annals of Nuclear Energy, 119(9), 342-351.

[23] Tao X.M., Li C.X., Shen W., et al. (2018) The SVDD Classifier for Unbalanced Data Based on Density-Sensitive and Maximum Soft Margin. Acta Electronica Sinica, 46(11), 2725-2732.

[24] Wan S.T., Dou L.J., Li C. (2018) Fault feature extraction and classification of high voltage circuit breakers based on VMD and sample entropy. Journal of Vibration and Shock, 37(20), 32-38.

[25] Zhang W.T., Du J.Q., Wang B.W. (2018) Industrial Process Fault Detection Based on Wavelet De-noise and KECA. Control and Instruments in Chemical Industry, 45(7), 522536. 